\title{
ARTICLE OPEN \\ Highly compliant planar Hall effect sensor with sub 200 nT sensitivity
}

\author{
Pablo Nicolás Granell ${ }^{1,2,3}$, Guoliang Wang ${ }^{1}$, Gilbert Santiago Cañon Bermudez ${ }^{1}$, Tobias Kosub ${ }^{1}$, Federico Golmar ${ }^{3,4}$, Laura Steren ${ }^{4,5,6}$, \\ Jürgen Fassbender ${ }^{1}$ and Denys Makarov (iD)
}

Being a facet of flexible electronics, mechanically reshapeable magnetic field sensorics enable novel device ideas for soft robotics, interactive devices for virtual- and augmented reality and point of care diagnostics. These applications demand mechanically compliant yet robust sensor devices revealing high sensitivity to small magnetic fields. To push the detection limit of highly compliant and linear magnetic field sensors to be in the sub- $\mu$ T range, we explore a new fundamental concept for magnetic field sensing, namely the planar Hall effect in magnetic thin films. With their remarkable bendability down to $1 \mathrm{~mm}$, these compliant planar Hall effect sensors allow for an efficient detection of magnetic fields as small as $200 \mathrm{nT}$ with a limit of detection of $20 \mathrm{nT}$. We demonstrate the application potential of these devices as a direction (angle) as well as proximity (distance) sensors of tiny magnetic fields emanating from magnetically functionalized objects. With their intrinsic linearity and simplicity of fabrication, these compliant planar Hall effect sensors have the potential to become a standard solution for low field applications of shapeable magnetoelectronics in point of care applications and on-skin interactive electronics.

npj Flexible Electronics (2019)3:3; https://doi.org/10.1038/s41528-018-0046-9

\section{INTRODUCTION}

The field of flexible electronics flourished over recent years with numerous fundamental discoveries ${ }^{1-4}$ which enabled exciting device concepts ranging from flexible displays $s^{5,6}$ and batteries ${ }^{7,8}$ to implantable devices, ${ }^{9-13}$ just to name a few. Their success relies on this novel formulation of electronics being compliant, wearable, and matching the mechanics of biological tissues. ${ }^{14,15}$ For each of these applications specific actuating ${ }^{16,17}$ and sensing elements are required. In the latter case, diverse types of mechanically reshapeable sensors have been reported, which can detect mechanical, ${ }^{18,19}$ optical, ${ }^{20}$ thermal $^{21,22}$, and bioelectric $^{23,24}$ stimuli, among others.

Flexible magnetic field sensors have been applied for detecting the presence of magnetic fields of magnetically functionalized objects, ${ }^{25-27}$ for proximity detection in novel touchless humanmachine interaction concepts or for augmented and virtual reality applications $^{28-31}$ (Supporting Table 1). State of the art flexible magnetoelectronics operate typically with magnetic fields in the $\mathrm{mT}$ range, which are easily realizable using small permanent magnets. ${ }^{32}$ Recent works report on flexible magnetic field sensors sensitive to the geomagnetic field, which is of $50 \mu \mathrm{T}$ only. ${ }^{33-36}$ While flexible, the mechanical performance of the reported devices does not meet the requirements needed for the field of wearable or on-skin electronics. Therefore, although highly demanded for various applications ranging from point of care diagnostics to biomedical magnetic field detection, ${ }^{37,38}$ there are no highly compliant magnetic field sensors with sub- $\mu$ T sensitivity. To enable such a high sensitivity in a mechanically compliant form factor, a new fundamental effect towards magnetic field sensing should be introduced in the field of shapeable magnetoelectronics. In this respect, magnetic field sensors based on the planar Hall effect (PHE) are particularly interesting for sensing weak magnetic fields (lower than the geomagnetic field), because they are intrinsically linear around zero field and show great sensitivity ${ }^{39}$ (Supporting Table 2). Furthermore, these sensors are metal-based and can be easily integrated in an imperceptible electronics platform as needed to assure for superior mechanical performance. $^{40-42}$

Here, we report a highly compliant PHE sensor capable of detecting magnetic fields in the range of sub $200 \mathrm{nT}$ with a limit of detection of $20 \mathrm{nT}$. Owing to the fabrication on mechanically imperceptible polymeric foils with a thickness of $6 \mu \mathrm{m}$ only, the sensor is bendable to a radius of $1 \mathrm{~mm}$ without any degradation of its electrical resistance. This newly explored sensing mechanism based on the planar Hall effect, allows for an extremely simple sensor design consisting of one magnetic layer (Permalloy) and one contact layer. The sensor shows excellent bending performance with $0.3 \%$ resistance variation after more than 150 bending cycles. We report a maximum sensitivity of $0.86 \mathrm{~V} / \mathrm{T}$ in the linear range of $\pm 50 \mu \mathrm{T}$, which makes these sensors sensitive to magnetic fields down to $20 \mathrm{nT}$. Although prepared on ultrathin polymeric foils, the sensitivity of these sensors meets the state of the art values reported for their rigid counterparts. ${ }^{43-45}$ We showcase the performance of the sensor for two application examples in angle and proximity detection.

\footnotetext{
${ }^{1}$ Helmholtz-Zentrum Dresden-Rossendorf e.V., Institute of Ion Beam Physics and Materials Research, Bautzner Landstrasse 400,01328 Dresden, Germany; ${ }^{2}$ Instituto Nacional de Tecnología Industrial (INTI), Centro de Micro- y Nanoelectrónica del Bicentenario (CMNB), Av. Gral Paz 5445, B1650KNA San Martín, Buenos Aires, Argentina; ${ }^{3}$ Escuela de Ciencia y Tecnología, UNSAM, Campus Miguelete, B1650KNA San Martín, Buenos Aires, Argentina; ${ }^{4}$ Consejo Nacional de Investigaciones Científicas y Técnicas, C1425FQB Ciudad

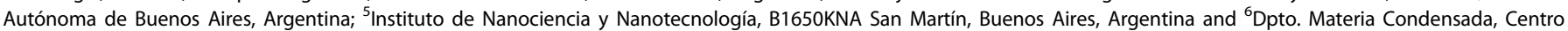
Atómico Constituyentes, B1650 San Martín, Buenos Aires, Argentina

Correspondence: Pablo Nicolás. Granell (pgranell@inti.gob.ar) or Denys Makarov (d.makarov@hzdr.de)
}

Received: 13 September 2018 Accepted: 16 December 2018

Published online: 01 February 2019 
(a) Prepare sensing layer Prepare contacts Device delamination
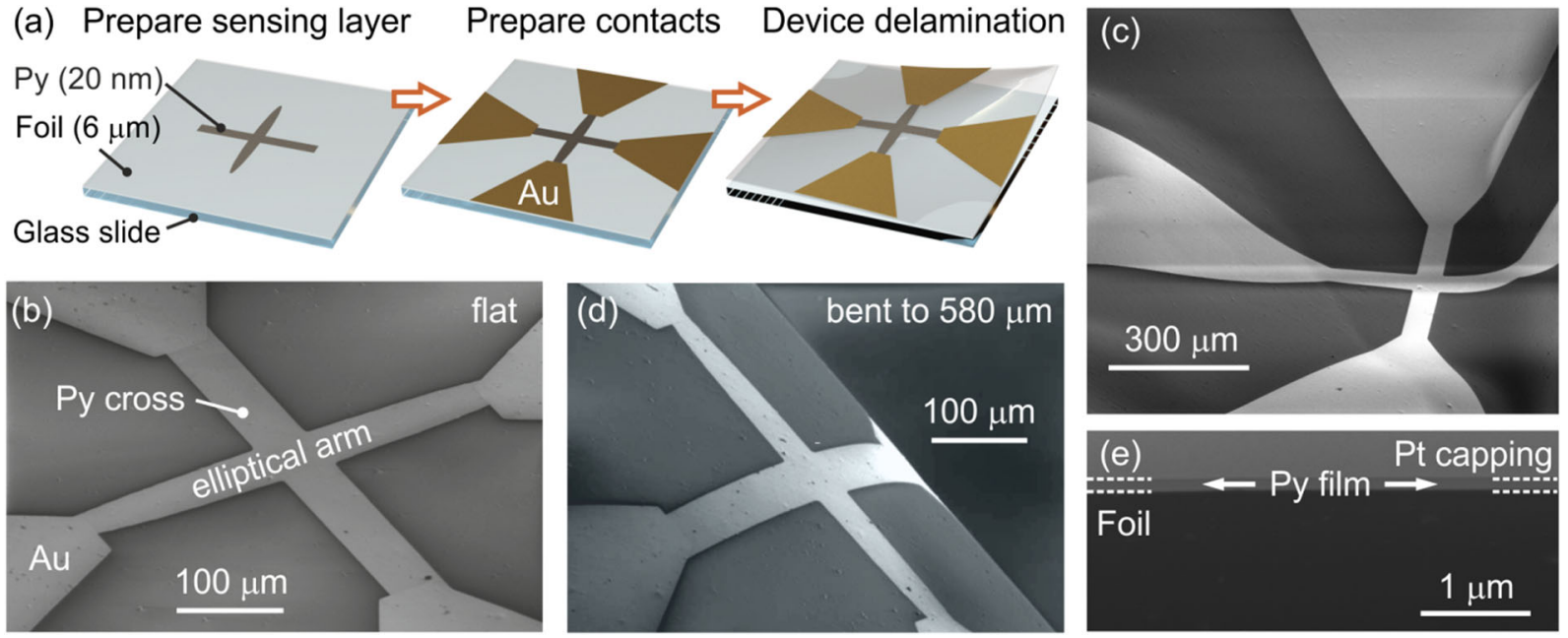

Fig. 1 Fabrication of highly compliant PHE sensors. a Schematics on the fabrication process. An ultrathin polymeric foil is attached to a supporting glass substrate. Optionally, an SU-8 smoothing layer can be added before the first patterning step. The magnetic sensing layer is prepared by photolithography and e-beam evaporation. Contact lines are realized in subsequent lithography and evaporation steps. Finally, the device is detached from the supporting glass slide. SEM imaging of a compliant PHE sensor in a planar $\mathbf{b}$, biaxially bent $\mathbf{c}$, and uniaxially bent $\mathbf{d}$ states. e SEM image showing a cross-section of the sample prepared using FIB milling

\section{RESULTS AND DISCUSSION}

Fabrication

The sensor is realized by microfabricating Permalloy (Py) Hall crosses with a thickness of $20 \mathrm{~nm}$ on $6-\mu \mathrm{m}$-thick PET foils (Fig. 1a). Optionally, a 2- $\mathrm{mm}$-thick SU-8 layer can be added to reduce the surface roughness of the PET foil. To improve the sensor performance, each stripe of the Hall cross is prepared with a high aspect ratio of 10:1 to induce a preferred magnetization axis of the Py structure by shape anisotropy. This is further facilitated by patterning an elliptically-shaped stripe (Fig. 1b) instead of a rectangular one. ${ }^{46}$ As the second and final fabrication step, electrical contacts are prepared to interface the Hall cross with the outside electronics. The integrity of the layer stack upon mechanical deformations is characterized by scanning electron microscopy (SEM) imaging (Fig. 1c, d). In addition to the top-view images, the sample cross-section is investigated upon Focused lon Beam (FIB) milling (Fig. 1e), revealing a firm adhesion of the metallic layer to a polymeric foil.

\section{Magnetoelectrical characterization}

To define the favorable magnetic state of the sensor, we applied a magnetic field parallel to the elliptical arm of the Hall cross, performing a magnetic field sweep between $\pm 3 \mathrm{mT}$. This field is sufficient to saturate the elliptically shaped Py stripe. After this standard initialization process, ${ }^{43}$ we determine the sensitivity axis of the sensors to be for the fields applied parallel to the elliptical axis. Figure 2 shows the magnetoresistive response of the sensor in the flat state and bent to a radius of $1 \mathrm{~mm}$. In the flat state, the sensor showed a maximum sensitivity of $0.86 \mathrm{~V} / \mathrm{T}$ when biased with a $5 \mathrm{~mA}$ direct current (DC). The actual measurement of the magnetic field strength experimentally detected by the sensor is $<200 \mathrm{nT}$ for the flat sensor (error bars shown in Fig. 2a correspond to the standard deviation of $200 \mathrm{nT}$ ). Even when bent to $1 \mathrm{~mm}$, the sensors still reveal a remarkably high sensitivity of $0.63 \mathrm{~V} / \mathrm{T}$. We relate the observed change in the sensitivity after bending to the modification of the magnetic domain pattern due to magnetostrictive effects. To assure a direct comparison of the measurements of the same sensor between the bent and flat states, we did not reinitialize the magnetic state of the sensor between the measurements in the flat and bent states. The change of the magnetic domain pattern in a magnetic thin film upon bending was investigated elsewhere. ${ }^{47-49}$
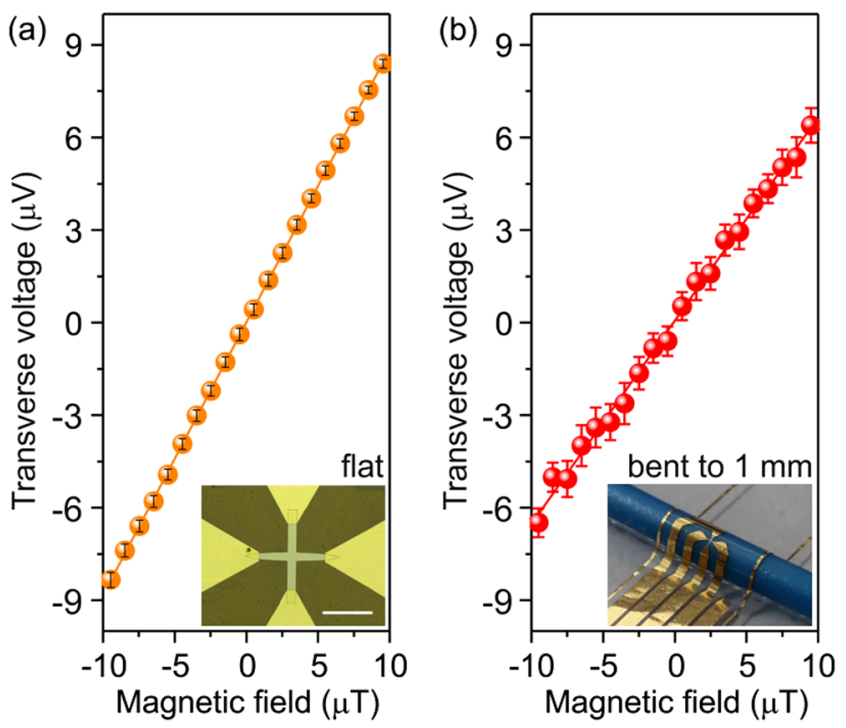

Fig. 2 Magnetoelectrical characterization in the linear range. Transverse voltage (planar Hall voltage) measured for a compliant PHE sensor $\mathbf{a}$ in the flat state (average of 5 measurements) and $\mathbf{b}$ bent to a radius of $1 \mathrm{~mm}$ (average of 7 measurements). The scale bar in panel a corresponds to $300 \mu \mathrm{m}$. Solid lines are linear fits to the experimental data points. Error bars calculated from standard deviation of measurements

Ultimately, the sensitivity of the sensors, namely its ability to measure the smallest fields, is determined by its noise. One of the great advantages of the metal-based PHE sensors is their small resistance, which allows for a very low intrinsic sensor noise. As our compliant PHE sensors have typical resistances of about $100 \Omega$, their intrinsic thermal noise (Johnson noise) is $\sim 1.3 \mathrm{nV} / \mathrm{Hz}^{\mathbf{1 / 2}}$ (equivalent magnetic field noise of $1.5 \mathrm{nT} / \mathrm{Hz}^{1 / 2}$ ). This remarkably low noise figure is compromised by the noise of the read-out electronics. In our experiments, we used electronics with a white noise density of $55 \mathrm{nV} / \mathrm{Hz}^{1 / 2}$. Considering the maximum integration time of $10 \mathrm{~s}$ for the compliant PHE sensor in a flat arrangement (corner frequency of $0.1 \mathrm{~Hz}$ in the power spectral density plot; Supporting information), the sensor would be able to discriminate fields down to $20 \mathrm{nT}$ (limit of detection). We note that 

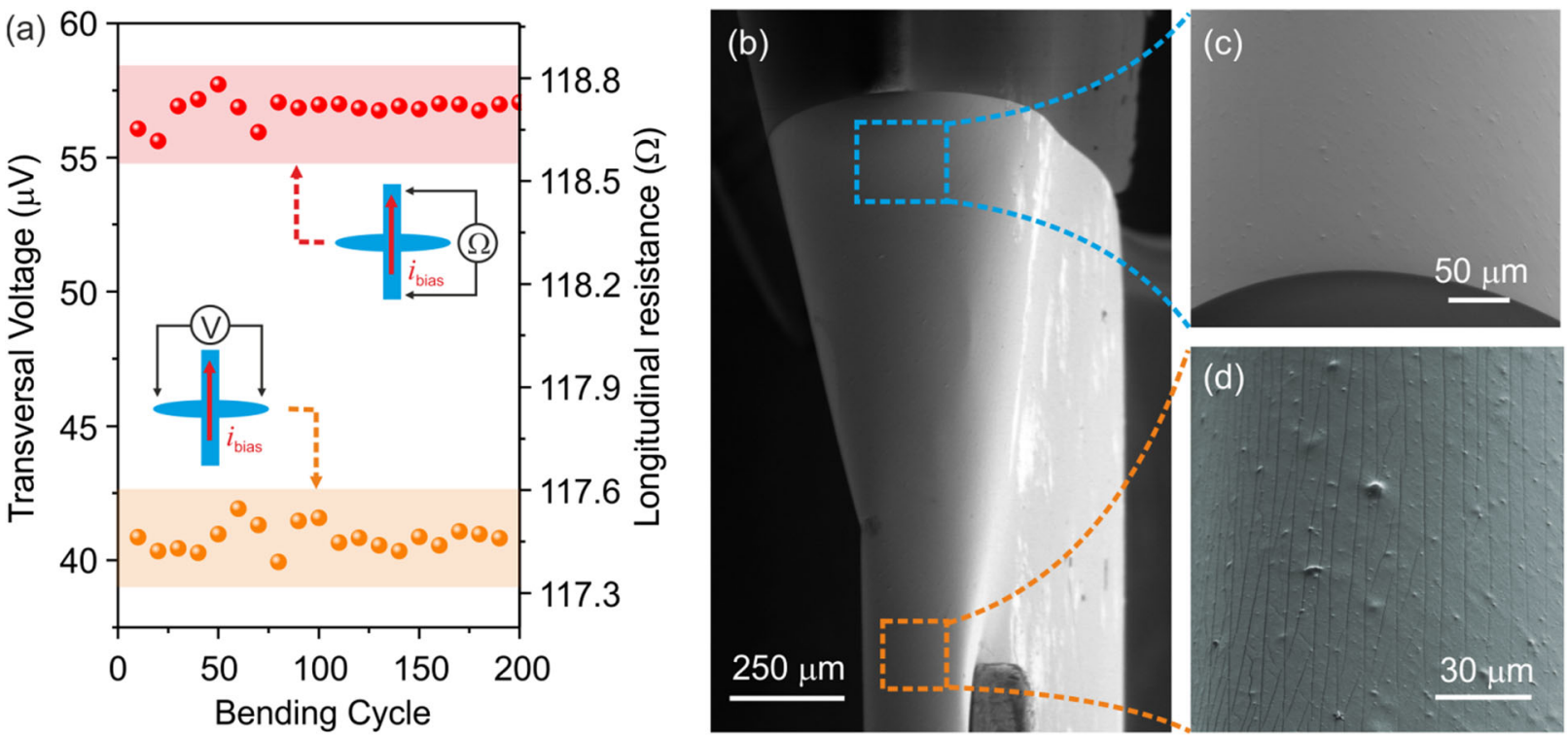

Fig. 3 Mechanical integrity tests. a Dynamic cycling of a compliant PHE sensor. The top dataset represents the measured longitudinal resistance along the bending direction. The bottom dataset shows the measured transversal voltage (Hall voltage) perpendicular to the bending direction. $\mathbf{b}$ SEM images of a bent sensor. The radius of curvature is gradually changing from about 100-300 $\mu \mathrm{m}$. The close-up images of the regions indicated in panel $\mathbf{b}$ are shown for the case of the bending radius of $\mathbf{c} 290 \mu \mathrm{m}$ and $\mathbf{d} 110 \mu \mathrm{m}$

this number is not generic as it is strongly dependent on the readout electronics. For instance, the use of a low noise electronics (typical white noise density of low noise electronics is $15 \mathrm{nV} / \mathrm{Hz}^{\mathbf{1 / 2}}$ ) would boost the limit of detection of a compliant PHE sensor to $10 \mathrm{nT}$.

\section{Mechanical performance}

The key requirement for compliant sensors is that their resistance remains essentially invariant upon mechanical deformations. We carried out extensive mechanical testing of the compliant PHE sensors in a cycling bending apparatus (Figure S2). The sensor was bent for more than 150 times between $4 \mathrm{~mm}$ and $2.4 \mathrm{~mm}$ bending radii (Movie S1). The electrical resistance parallel to the bending direction was recorded at the initial $(4 \mathrm{~mm})$ and final $(2.4 \mathrm{~mm})$ positions. Figure $3 a$ shows the evolution of the sample resistance at the final bending position $(2.4 \mathrm{~mm})$, showing a variation of $0.3 \%$ only during the entire cycling test. With this, we have realized a strain invariant yet mechanically compliant magnetic field sensor relying on the planar Hall effect.

Furthermore, we recorded the transverse voltage of the sensor during the cyclic bending experiment. The results are shown in Fig. $3 a$ for the case when the sensor is bent to a radius of $2.4 \mathrm{~mm}$. Only a small variation of the transversal voltage upon cycling is observed. The voltage variation in this dynamic bending test is attributed to the change of the voltage offset, which is not related to any magnetic effect but to the sensor contact geometry. Still, no degradation of the sensor output is found in the data.

The critical bending radius of the functional Py layer was characterized using SEM. We folded a sensor device to different bending radii down to about $100 \mu \mathrm{m}$ (Fig. 3b). The examination of the morphology of the bent sensor reveals that the surface remains continuous without evident film fracturing if the bending radius exceeds $290 \mu \mathrm{m}$ (Fig. 3c). For smaller bending radii, cracks start to evolve as demonstrated for the case when the bending radius is $110 \mu \mathrm{m}$ (Fig. $3 \mathrm{~d}$ ). These experimental results are in a good quantitative agreement with analytical estimations revealing a critical bending radius of $265 \mu \mathrm{m}$. The corresponding strain experienced by the Permalloy film is calculated to be $1.7 \times 10^{-1}$ for a bending radius of $290 \mu \mathrm{m}$ and $4.5 \times 10^{-1}$ for a bending radius of $110 \mu \mathrm{m}$. We note that this remarkable mechanical performance is achieved due to the use of ultrathin polymeric foils. For comparison, the use of standard $100-\mu m$-thick polymeric substrates would result in a critical bending radius of about 1 order of magnitude higher ( $5 \mathrm{~mm}$, as typically observed experimentally ${ }^{34}$ ).

Compliant PHE sensors for detecting magnetic stray fields

Due to their excellent mechanical properties, the sensor devices can be bent around curved objects and provide information of tiny magnetic fields emanating from them. In this way, the sensor can be placed in close proximity to a signal source. For example, it could be implemented to detect magnetic functionalized objects flowing in a fluidic channel or the stray magnetic field generated by a current-carrying wire. As a proof of principle, the performance of compliant PHE sensors was evaluated by measuring the magnetic field generated by a pulsing DC current in a stranded copper wire with an outer diameter of $2 \mathrm{~mm}$ (nominal thickness of the insulation is $0.45 \mathrm{~mm}$ ). For the measurement, the sensor was wrapped around the wire to be in the closest proximity to the source of magnetic field (Fig. 4a). To determine the magnetic field around the wire, we carried out finite element simulations (Finite Element Method Magnetics v4.2) taking into account the stranded structure of the inner copper threads (Fig. 4b). It was found out that the field on the surface of the wire is only about $20 \mu \mathrm{T}$ when a DC current of $100 \mathrm{~mA}$ is supplied to it. We note that this field is similar to the one typically obtained in a magneto-fluidic experiments. $^{25}$

For the experiment, we recorded the PHE voltage while manually switching the DC current circulating through the wire ON and OFF (Movie S2). A clear jump in the readout voltage is produced when the current is turned on (Fig. 4d). Furthermore, the linearity of our sensor is preserved, as seen by the magnitude of the voltage change when the current is reversed. This confirms that the magnetic field effects are being measured instead of a temperature variation upon Joule heating of the wire. We note that the performance of the compliant PHE sensors is at least 10 times better compared to the conventional bulky and rigid clamp meters applied for current sensing. ${ }^{50}$ 

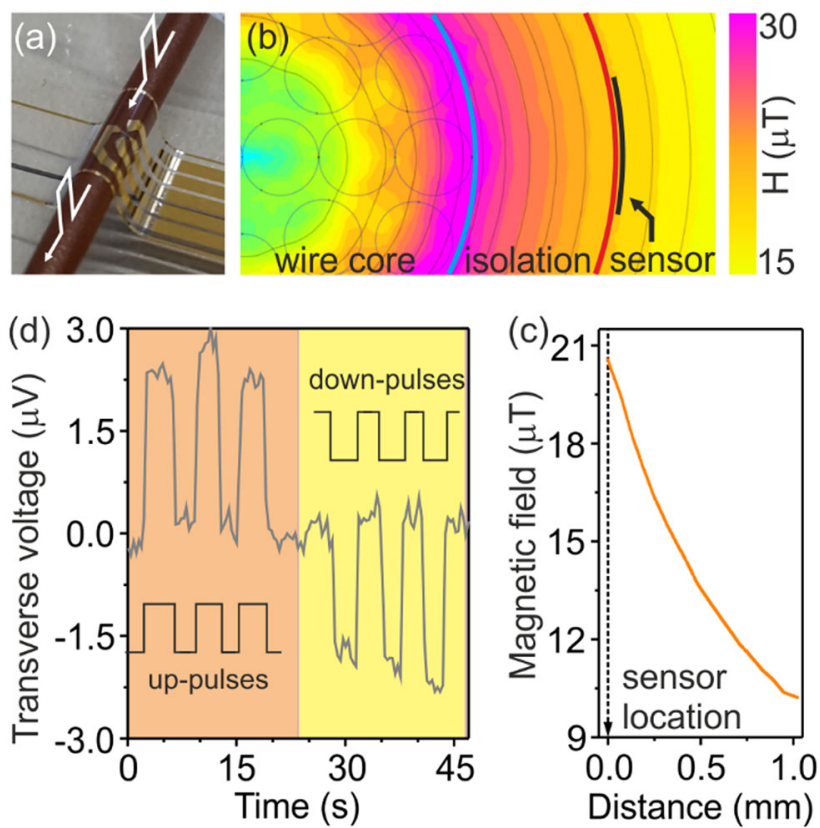

Fig. 4 Detection of magnetic stray fields. a A compliant PHE sensor is wrapped around a copper wire with a radius of $1 \mathrm{~mm}$. White arrows represent schematically DC current pulses in the wire. b Finite element simulations reveal a density plot of the magnetic flux, generated by the wire upon applying DC current pulses of $100 \mathrm{~mA}$. C A profile of the magnetic flux density from the wire surface indicating an intensity of $20.4 \mu \mathrm{T}$ at the sensor location. $\mathbf{d}$ Transverse voltage measured by the sensor, clearly separating the incoming current pulses of different polarity

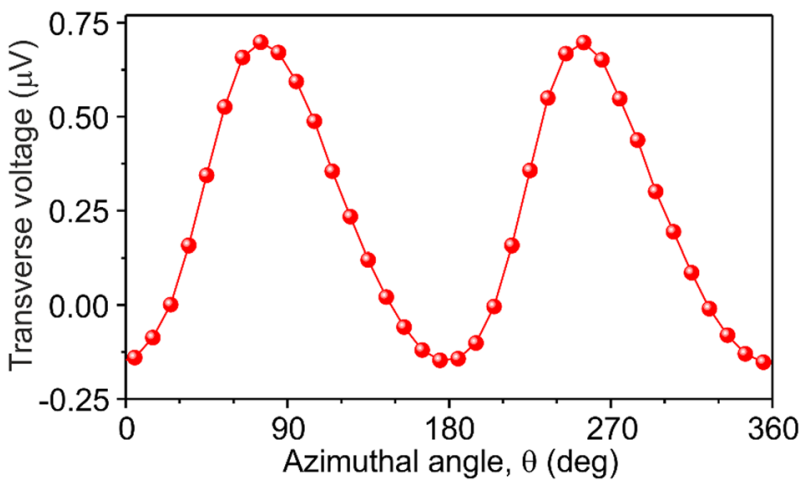

Fig. 5 Angular sensor application. The angular dependence of the transverse voltage was measured using a compliant PHE sensor exposed to a saturating field of $4 \mathrm{mT}$. Solid line is a guide to the eye

Compliant PHE sensors as angular sensors

We demonstrated the performance of the compliant PHE sensor in the linear regime to detect weak magnetic fields. Still, the PHE sensors can also be used for the detection of an angle. In this case, we take advantage of the angular dependence of the PHE sensor output with the relative orientation of the magnetization with respect to the biasing current. We evaluated the angular dependence of the compliant PHE sensors by applying a saturating field of $4 \mathrm{mT}$. A $\sin (2 \theta)$ angular dependence is expected according to the off-diagonal terms of the resistivity tensor for anisotropic ferromagnetic materials (detailed calculations in $\mathrm{SI}$ ). In this demonstrator, the sample was placed on a rotating stage in the center of a pair of Helmholtz coils (Figure S4) and the PHE voltage was measured. Figure 5 shows the measured transverse voltage as a function of the angle between the sensor axis and the applied field (azimuthal angle) for a sample bent to a radius of $1 \mathrm{~mm}$. The detected response verifies the $\sin (2 \theta)$ angular dependence. When used within its linear range, the sensor could be used as an angular sensor to provide orientation information in soft robotics applications, benefiting from the high conformability of the ultrathin polymeric substrate.

Here, we applied a new fundamental physical principle of magnetic field sensing for shapeable magnetoelectronics. By doing this, we realized a compliant yet high-performance magnetic field sensor relying on the planar Hall effect in magnetic thin films. Even when prepared on mechanically imperceptible 6$\mu \mathrm{m}$-thick polymeric foils, the sensor elements revealed a remarkable sensitivity to magnetic fields of $0.86 \mathrm{~V} / \mathrm{T}$, which allows us to detect magnetic fields as small as $200 \mathrm{nT}$ in the flat state. Owing to the ultra-thin supporting layer, the compliant planar Hall effect sensors do not reveal any sign of degradation in a cyclic bending experiment with a tiny resistance variation of $<0.3 \%$ when bending the devices down to a radius of $2.4 \mathrm{~mm}$. The application potential of the device is showcased in two examples of an angle and proximity sensors. For the latter, we demonstrate that the compliant PHE sensor is able to detect small magnetic stray fields of magnetically functionalized objects as needed for conventional metrology as well as point of care diagnostics. High sensitivity of the prepared sensing devices at field ranges lower than the geomagnetic field combined with a remarkable simplicity of fabrication, is a step forward in the realization of cost efficient flexible magnetoelectronic devices, with possible application in soft robotics, interactive devices for virtual- and augmented reality and point of care platforms for the detection of magnetic objects.

\section{MATERIALS AND METHODS}

\section{Substrate preparation}

For preparing the compliant PHE sensors, we used commercial polyethylenterephthalat (PET) foils (Chemplex Inc., USA) with a nominal thickness of $6 \mu \mathrm{m}$. The foils were attached to a rigid support, i.e. glass slides, to allow for a convenient manipulation upon lithography and metal evaporation steps. We prepared the samples in two ways: by direct patterning onto PET foils or by adding an extra smoothing layer to reduce the surface roughness. For the latter case, we spin coated a layer of epoxybased photoresist SU-8 (Microchem Corp., USA) for $30 \mathrm{~s}$ at $2000 \mathrm{rpm}$, resulting in a nominal thickness of $2 \mu \mathrm{m}$. Then, the samples were soft baked ( $1 \mathrm{~min}$ at $65^{\circ} \mathrm{C}$ followed by $3 \mathrm{~min}$ at $95^{\circ} \mathrm{C}$ ) and exposed to a UV light (365 $\mathrm{nm}$ wavelength) for $10 \mathrm{~min}$. Finally, the resist was cured for $10 \mathrm{~min}$ at $150^{\circ} \mathrm{C}$. The overall thickness of the flexible support was $8 \mu \mathrm{m}$ including the SU-8 layer.

\section{Sensor fabrication}

Permalloy structures with a Hall cross geometry were patterned in a first lithography step. We spin coated a layer of adhesion promoter TI Prime (Microchemicals $\mathrm{GmbH}$ ) at $4000 \mathrm{rpm}$ for $30 \mathrm{~s}$, followed by soft bake for $2 \mathrm{~min}$ at $120^{\circ} \mathrm{C}$. Then, image reversal photoresist AZ5214E (Microchemicals $\mathrm{GmbH}$ ) was spin coated for $30 \mathrm{~s}$ at $4000 \mathrm{rpm}$. The samples were soft baked for $50 \mathrm{~s}$ at $110^{\circ} \mathrm{C}$ and then exposed with a UV laser writer machine (Heidelberg DWL66). After the first exposure, image reversal bake followed, heating the samples for $2 \mathrm{~min}$ at $120^{\circ} \mathrm{C}$. Once the samples cooled down, they were exposed for the second time to a UV light ( $365 \mathrm{~nm}$ wavelength) for 5 min. Finally, the samples were developed in AZ351B (Microchemicals $\mathrm{GmbH}$ ) with a dilution of 1:4 in deionized (DI) water. The development time was $40 \mathrm{~s}$ with minimal agitation. After patterning, a 20-nm-thick layer of Permalloy $\left(\mathrm{Ni}_{80} \mathrm{Fe}_{20}\right)$ was deposited by electron beam evaporation (pressure: $8 \times 10^{-8} \mathrm{mbar}$; deposition rate: $0.13 \mathrm{~nm} / \mathrm{s}$ ), followed by the evaporation of a protecting 3-nm-thick Au capping layer (pressure: $6.2 \times$ $10^{-8} \mathrm{mbar}$; deposition rate: $0.24 \mathrm{~nm} / \mathrm{s}$ ). In the second fabrication step, we evaporate and pattern electrical contact pads consisting of $[\mathrm{Au}(100 \mathrm{~nm}) / \mathrm{Ti}$ $(3 \mathrm{~nm})$ ] bilayers (pressure: $2.2 \times 10^{-8} \mathrm{mbar}(\mathrm{Ti})$ and $5.5 \times 10^{-8} \mathrm{mbar}(\mathrm{Au})$; deposition rate: $0.02 \mathrm{~nm} / \mathrm{s}(\mathrm{Ti})$ and $0.55 \mathrm{~nm} / \mathrm{s}(\mathrm{Au})$ ). 


\section{Magnetoelectrical chararacterization}

To evaluate the magnetotransport properties of the fabricated sensors, we placed a sample in the center of a pair of Hemholtz coils, which were supplied with a DC current using a Keysight B2902A precision sourcemeter. The magnetic field produced by the Helmholtz coils was calibrated using a Gaussmeter (Goudsmith Magnetic Systems, Netherlands). A Keysight B2902A sourcemeter was used to set a DC current bias of $5 \mathrm{~mA}$ to the sensor for magnetotransport measurements. A Keysight $34461 \mathrm{~A}$ multimeter was used for DC voltage readout. For angular dependence measurements, we placed the sample on a rotating stage controlled by a stepper motor. Full hysteresis loops were also measured for a field range of $\pm 3 \mathrm{mT}$ and different bending radii (Figure S3).

\section{Mechanical performance}

A custom-built cyclic bending machine was employed to dynamically bend the sensor and simultaneously measure its resistance. The compliant PET foils were adapted to fit into a commercial Flexible Printed Circuit (FPC) socket (Figure S2b) to ensure mechanically robust contacts during cycling. The socket was soldered to a printed circuit board (PCB), which was attached to the moving part of the bending apparatus. On the stationary side, we fixed a small plastic press to hold the other side of the sample (Figure S2a).

\section{Calculations of critical bending radius}

The smallest bending radius tolerable by a Permalloy thin film can be estimated relying on the formalism developed in: ${ }^{51}$

$\varepsilon_{\text {top }}=\frac{\left(d_{f}+d_{s}\right)}{2 R} \frac{\left(1+2 \eta+x \eta^{2}\right)}{(1+\eta)(1+x \eta)}$

Where $\eta=d_{f} / d_{s}$ and $X=Y_{f} / Y_{s} . d_{f}$ is the total thickness of the active metallic layers $\left(20 \mathrm{~nm}\right.$ Py and $3 \mathrm{~nm} \mathrm{Au}$ ) and $d_{s}$ is the substrate thickness (6$\mu \mathrm{m}$-thick PET). $Y_{f}$ and $Y_{s}$ are the Young's moduli of the metallic films and substrate, respectively. $\varepsilon_{\text {top }}$ is the surface strain when the structure is bent to a radius $R$. In ref. ${ }^{52}$ it was found that the critical strain before the first fractures in 60-nm-thick Py film are observed, is in the range of 0.85 to $1 \%$. Using this data with Equation 1, we estimated that the critical radius for our device is about $265 \mu \mathrm{m}$.

\section{Electrical output of planar Hall effect sensors}

Referring to the geometry in Figure S1, a uniform current $I_{x}$ circulating through a bar-shaped ferromagnetic thin film of thickness $t$ will produce a transverse voltage $V_{y}$ given by: ${ }^{53}$

$V_{y}=\frac{I_{x} \Delta \rho \sin (2 \theta)}{2 t}$

With $\Delta \rho=\rho_{\|}-\rho_{\perp}$, where $\rho_{\|}$and $\rho_{\perp}$ are the resistivities parallel and perpendicular to the magnetization direction. A magnetic thin film in a single domain state is assumed with in-plane magnetization along the unit vector $\hat{M}=(\cos \theta, \sin \theta)$. This expression is strictly valid for a Hall bar with infinitesimal voltage probes, but it reflects the expected angular dependence for our sensors.

\section{ACKNOWLEDGEMENTS}

We thank Bernd Scheumann, Rainer Kaltofen, and Dr. Jens Ingolf Mönch (all HZDR) for the deposition of metal layer stacks. Support by the Structural Characterization Facilities Rossendorf at the Ion Beam Center (IBC) at the HZDR as well as the SEM/FIB characterization facility at the INTI is greatly appreciated. The project is funded in part via the German Academic Exchange Service (DAAD) and the Ministry of Education of Argentina for scholarship "Country-related cooperation programme with Argentina ALEARG short term scholarship, 2017 (57352687)" and German Research Foundation (DFG) Grant MA 5144/9-1.

\section{AUTHOR CONTRIBUTIONS}

P.N.G. and D.M. formulated the task. P.N.G. carried out experimental work with the contribution from G.W., T.K., and G.S.C.B.; P.N.G. performed electromagnetic simulations with the contribution from F.G. and L.S.; P.N.G., D.M., T.K., and G.S.C.B. analyzed the data with contributions from J.F., L.S., and F.G.; The manuscript was written by D.M., P.N.G., and T.K. with contributions from J.F., G.W., L.S., F.G., and G.S.C. B. All authors have given approval to the final version of the manuscript.

\section{ADDITIONAL INFORMATION}

Supplementary information accompanies the paper on the npj Flexible Electronics website (https://doi.org/10.1038/s41528-018-0046-9).

Competing interests: The authors declare no competing interests.

\section{REFERENCES}

1. Bauer, S. Sophisticated skin: flexible electronics. Nat. Mater. 12, 871-872 (2013).

2. Bauer, S. et al. 25th anniversary article: a soft future: from robots and sensor skin to energy harvesters. Adv. Mater. 26, 149-162 (2014).

3. Lipomi, D. J. \& Bao, Z. Stretchable and ultraflexible organic electronics. MRS Bull. 42, 93-97 (2017).

4. Yu, K. J., Yan, Z., Han, M. \& Rogers, J. A. Inorganic semiconducting materials for flexible and stretchable electronics. npj Flexible Electronics 1, 4 (2017).

5. White, M. S. et al. Ultrathin, highly flexible and stretchable PLEDs. Nat. Photonics 7, 811-816 (2013).

6. Choi, M. K. et al. Extremely vivid, highly transparent, and ultrathin quantum dot light-emitting diodes. Adv. Mater. 30, 1703279 (2018).

7. Ha, S. H., Shin, K. H., Park, H. W. \& Lee, Y. J. Flexible lithium-ion batteries with high areal capacity enabled by smart conductive textiles. Small. 1703418 (2018). https://doi.org/10.1002/smll.201703418

8. Liu, Q.-C., Xu, J.-J., Xu, D. \& Zhang, X.-B. Flexible lithium-oxygen battery based on a recoverable cathode. Nat. Commun. 6, 7892 (2015).

9. Fang, H. et al. Ultrathin, transferred layers of thermally grown silicon dioxide as biofluid barriers for biointegrated flexible electronic systems. Proc. Natl Acad. Sci. USA 113, 11682-11687 (2016).

10. Shin, G. et al. Flexible near-field wireless optoelectronics as subdermal implants for broad applications in optogenetics. Neuron 93, 509-521.e3 (2017)

11. $\mathrm{Yu}, \mathrm{K}$. J. et al. Bioresorbable silicon electronics for transient spatiotemporal mapping of electrical activity from the cerebral cortex. Nat. Mater. 15, 782-791 (2016).

12. Park, S. I. et al. Soft, stretchable, fully implantable miniaturized optoelectronic systems for wireless optogenetics. Nat. Biotechnol. 33, 1280-1286 (2015).

13. Uguz, I. et al. A microfluidic ion pump for in vivo drug delivery. Adv. Mater. 29, 1701217 (2017).

14. Amjadi, M., Kyung, K.-U., Park, I. \& Sitti, M. Stretchable, skin-mountable, and wearable strain sensors and their potential applications: a review. Adv. Funct. Mater. 26, 1678-1698 (2016).

15. Miyamoto, A. et al. Inflammation-free, gas-permeable, lightweight, stretchable on-skin electronics with nanomeshes. Nat. Nanotechnol. 12, 907-913 (2017).

16. Gisby, T. A., O'Brien, B. M. \& Anderson, I. A. Self sensing feedback for dielectric elastomer actuators. Appl. Phys. Lett. 102, 193703 (2013).

17. Anderson, I. A., Gisby, T. A., McKay, T. G., O'Brien, B. M. \& Calius, E. P. Multifunctional dielectric elastomer artificial muscles for soft and smart machines. J. Appl. Phys. 112, 041101 (2012).

18. Yamada, T. et al. A stretchable carbon nanotube strain sensor for human-motion detection. Nat. Nanotechnol. 6, 296-301 (2011).

19. Ramuz, M., Tee, B. C.-K., Tok, J. B.-H. \& Bao, Z. Transparent, optical, pressuresensitive artificial skin for large-area stretchable electronics. Adv. Mater. 24, 3223-3227 (2012).

20. Ko, H. C. et al. A hemispherical electronic eye camera based on compressible silicon optoelectronics. Nature 454, 748-753 (2008).

21. Webb, R. C. et al. Ultrathin conformal devices for precise and continuous thermal characterization of human skin. Nat. Mater. 12, 938-944 (2013).

22. Drack, M. et al. An imperceptible plastic electronic wrap. Adv. Mater. 27, 34-40 (2015).

23. Graudejus, O., Yu, Z., Jones, J., Morrison, B. \& Wagner, S. Characterization of an elastically stretchable microelectrode array and its application to neural field potential recordings. J. Electrochem. Soc. 156, P85 (2009).

24. Kim, D.-H. et al. Materials for multifunctional balloon catheters with capabilities in cardiac electrophysiological mapping and ablation therapy. Nat. Mater. 10, 316-323 (2011).

25. Lin, G. et al. A highly flexible and compact magnetoresistive analytic device. Lab Chip 14, 4050-4058 (2014).

26. Melzer, M. et al. Elastic magnetic sensor with isotropic sensitivity for in-flow detection of magnetic objects. RSC Adv. 2, 2284 (2012)

27. Melzer, M. et al. Stretchable magnetoelectronics. Nano Lett. 11, 2522-2526 (2011).

28. Melzer, M. et al. Imperceptible magnetoelectronics. Nat. Commun. 6, 6080 (2015).

29. Melzer, M. et al. Wearable magnetic field sensors for flexible electronics. Adv. Mater. 27, 1274-1280 (2015).

30. Cañón Bermúdez, G. S. et al. Magnetosensitive e-skins with directional perception for augmented reality. Sci. Adv. 4, eaao2623 (2018). 
31. Münzenrieder, N. et al. Entirely flexible on-site conditioned magnetic sensorics. Adv. Electron. Mater. 2, 1600188 (2016).

32. Makarov, D., Melzer, M., Karnaushenko, D. \& Schmidt, O. G. Shapeable magnetoelectronics. Appl. Phys. Rev. 3, 011101 (2016).

33. Li, B., Kavaldzhiev, M. N. \& Kosel, J. Flexible magnetoimpedance sensor. J. Magn. Magn. Mater. 378, 499-505 (2015).

34. Wang, Z. et al. Highly sensitive flexible magnetic sensor based on anisotropic magnetoresistance effect. Adv. Mater. 28, 9370-9377 (2016).

35. Wang, Z., Shaygan, M., Otto, M., Schall, D. \& Neumaier, D. Flexible Hall sensors based on graphene. Nanoscale 8, 7683-7687 (2016).

36. Cañón Bermúdez, G. S. et al. Electronic-skin compasses for geomagnetic fielddriven artificial magnetoreception and interactive electronics. Nat. Electron. 1, 589-595 (2018).

37. Freitas, P. P. et al. Spintronic platforms for biomedical applications. Lab Chip 12, 546-557 (2012).

38. Lin, G., Makarov, D. \& Schmidt, O. G. Magnetic sensing platform technologies for biomedical applications. Lab Chip 17, 1884-1912 (2017).

39. Schuhl, A., Van Dau, F. N. \& Childress, J. R. Low-field magnetic sensors based on the planar Hall effect. Appl. Phys. Lett. 66, 2751-2753 (1995).

40. Kaltenbrunner, M. et al. An ultra-lightweight design for imperceptible plastic electronics. Nature 499, 458-463 (2013).

41. Salvatore, G. A. et al. Wafer-scale design of lightweight and transparent electronics that wraps around hairs. Nat. Commun. 5, 2982 (2014).

42. Someya, T., Bauer, S. \& Kaltenbrunner, M. Imperceptible organic electronics. MRS Bull. 42, 124-130 (2017).

43. Mor, V. et al. Planar Hall effect sensors with shape-induced effective single domain behavior. J. Appl. Phys. 111, 07E519 (2012).

44. Hung, T. Q. et al. Optimization of the multilayer structures for a high fieldsensitivity biochip sensor based on the planar hall effect. IEEE Trans. Magn. 45, 4518-4521 (2009).

45. Ejsing, L. et al. Planar Hall effect sensor for magnetic micro- and nanobead detection. Appl. Phys. Lett. 84, 4729-4731 (2004).
46. Telepinsky, Y. et al. Towards a six-state magnetic memory element. Appl. Phys. Lett. 108, 182401 (2016)

47. Karnaushenko, D. et al. Self-assembled on-chip-integrated giant magnetoimpedance sensorics. Adv. Mater. 27, 6582-6589 (2015).

48. Streubel, R. et al. Magnetic microstructure of rolled-up single-layer ferromagnetic nanomembranes. Adv. Mater. 26, 316-323 (2014).

49. Streubel, R. et al. Magnetically capped rolled-up nanomembranes. Nano Lett. 12, 3961-3966 (2012).

50. Fluke 325 Clamp Meter User's Manual. Available at: www.fluke.com.

51. Suo, Z., Ma, E. Y., Gleskova, H. \& Wagner, S. Mechanics of rollable and foldable film-on-foil electronics. Appl. Phys. Lett. 74, 1177-1179 (1999).

52. Wojciechowski, P. H. \& Mendolia, M. S. On the multiple fracture of low-elongation thin films deposited on high-elongation substrates. J. Vac. Sci. Technol. A 7, 1282-1288 (1989).

53. Henriksen, A. D. et al. Planar Hall effect bridge magnetic field sensors. Appl. Phys. Lett. 97, 013507 (2010).

(i) Open Access This article is licensed under a Creative Commons Attribution 4.0 International License, which permits use, sharing, adaptation, distribution and reproduction in any medium or format, as long as you give appropriate credit to the original author(s) and the source, provide a link to the Creative Commons license, and indicate if changes were made. The images or other third party material in this article are included in the article's Creative Commons license, unless indicated otherwise in a credit line to the material. If material is not included in the article's Creative Commons license and your intended use is not permitted by statutory regulation or exceeds the permitted use, you will need to obtain permission directly from the copyright holder. To view a copy of this license, visit http://creativecommons. org/licenses/by/4.0/.

(c) The Author(s) 2019 